Hemendra N. Shah $\cdot$ Sanjay Nabbar

\title{
Extensive genitourinary tuberculosis presenting as spontaneous vesico-vaginal fistula
}

Published online: 26 October 2004

(C) Springer-Verlag Berlin / Heidelberg 2004

\begin{abstract}
We report a case of extensive genitourinary tuberculosis presenting as spontaneous vesico-vaginal fistula.
\end{abstract}

Keywords Tuberculosis - Vesico-vaginal fistula · Thimble bladder

\section{Introduction}

Genitourinary tuberculosis is an important cause of morbidity in developing and developed countries. It is also known for its varying presentation with the symptoms and signs varying both in intensity and duration. We describe a case of extensive genitourinary tuberculosis in which there was complete destruction of the right kidney, stricture of the left terminal ureter and an autocystectomized bladder with spontaneous vesico-vaginal fistula. She was treated with right nephroureterectomy and ileal conduit urinary diversion.

\section{Case report}

A 54-year-old female patient presented with dribbling of urine per vaginam for 15 days. There were no other urinary symptoms. She had undergone an abdominal hysterectomy 8 years ago for multiple uterine fibroids. On examination, there was a Pfanensteil scar from a previous surgery, and her internal examination revealed induration in the anterior fornix. She had a serum creatinine of $1.7 \mathrm{mg} \%$. Her ultrasound examination showed a grossly hydronephrotic right kidney with a paper-thin cortex and a moderate left hydroureteronephrosis. The right kidney was non-visualised on intravenous urogram, and there was moderate left hydroureteronephrosis with a small capacity bladder (Fig. 1). On DTPA renal scan, the right kidney was contributing only $8 \%$ to the total renal function.

On cystoscopic examination, the bladder capacity was approximately $80 \mathrm{cc}$ and its mucosa was inflamed and oedematous. Ure-

\section{H. N. Shah $(-)$ S. Nabbar}

R.G. Stone Urological Research Institute,

14-A 21-A Road Ahimsa Marg Khar (W), 400052 Mumbai, India e-mail: drhemendrashah@yahoo.co.in

Tel.: +91-22-55443609

Fax: $+91-22-26462123$ teric orifices were not identified, and there was a $1-\mathrm{cm}$ fistulous opening on the posterior wall. Percutaneous nephrostomy was done for the solitary functioning hydronephrotic left kidney. Bladder mucosal biopsies revealed tubercular infection. After diversion, the patient was not passing urine perurethrally, and the dribbling per vaginam had significantly decreased. Her serum creatinine reduced to $1.3 \mathrm{mg} \%$. The patient was given anti-tubercular treatment for 6 weeks and explored for right nephroureterectomy with bladder augmentation and fistula closure. A CT scan done prior to exploration revealed a normally functioning left kidney and a destroyed hydronephrotic right kidney (Fig. 2). On pelvic CT scan, a small, thick-walled bladder was seen (Fig. 3). At the time of exploration, cystoscopy revealed a markedly contracted bladder with a capacity of $10 \mathrm{cc}$ and a leakage of irrigation fluid per vaginam. The patient underwent right nephroureterectomy with the left ureter opened into an ileal conduit. Postoperatively, the patient was on anti-tubercular treatment and is asymptomatic at the 3-month follow-up.

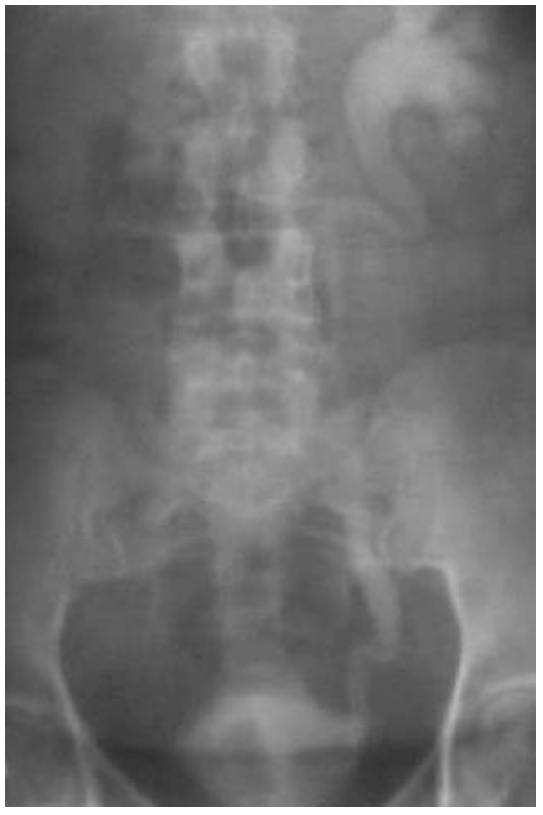

Fig. 1 Intravenous urogram showing non-visualised right kidney with left moderate hydroureteronephrosis and a small capacity bladder 


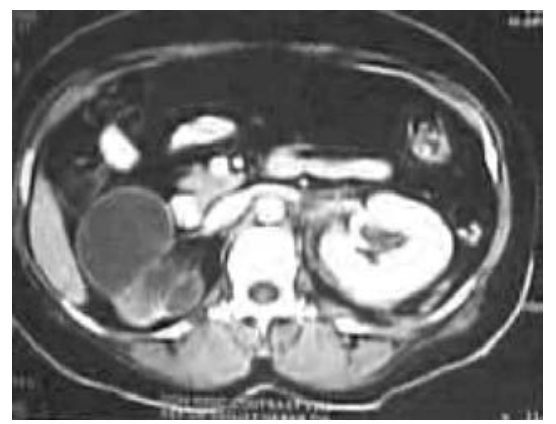

Fig. 2 CT scan of the abdomen with intravenous contrast showing normally functioning left kidney and destroyed hydronephrotic right kidney

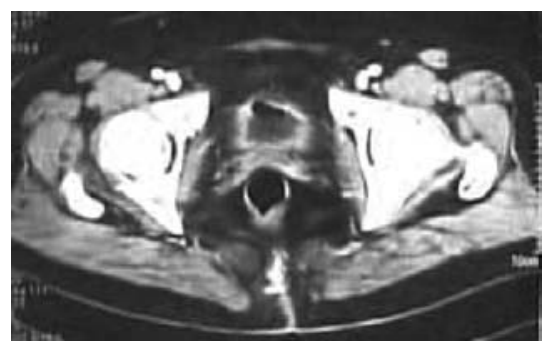

Fig. 3 CT scan of the pelvis showing a small, thick-walled bladder

\section{Discussion}

Genitourinary tuberculosis is still prevalent in tropical countries, and sometimes in advanced cases surgical reconstruction is required. This case represents a rare variety of genitourinary tuberculosis that presented with spontaneous vesico-vaginal fistula. Inspite of the extensive involvement of the genitourinary tract leading to tuberculous right autonephrectomy, thimble bladder and involvement of the left terminal ureter leading to hydroureteronephrosis, the patient had no symptoms attributable to her urinary tract. It was only after spontaneous fistulation in the vagina that the patient developed dribbling of urine per vaginam.

Thimble bladder is commonly seen in genitourinary tuberculosis, especially after initiation of antitubercular treatment and frequently requires surgical treatment, either augmentation cystoplasty or urinary diversion [1]. Our patient developed total destruction of the bladder over a period of 6 weeks after initiation of anti-tubercular treatment. A similar case of primary genitourinary tuberculosis associated with severe progressive scarring and destruction of the left kidney with a contracted bladder, persistent vesicoureteral reflux and bulbar urethral stricture in which scarring started after initiation of medical therapy has been reported [2]. The doctors recommended close supervision after initiation of therapy for genitourinary tuberculosis. In the present case, lack of distension of the bladder secondary to urinary diversion of the solitary functioning kidney would have contributed to the process of vesical destruction.

Tuberculous autonephrectomy is a well-described entity in which there is complete destruction of the kidney, usually silent and asymptomatic. Tubercular auto-cystectomy is also described in the literature; however, this entity is extremely rare [3]. Spontaneous vesico-vaginal fistula of tuberculous origin has been described $[4,5]$. Our patient had simultaneous tubercular auto-cystectomy and auto-nephrectomy with a spontaneous vesico-vaginal fistula.

Tuberculosis is known to have unusual presentations, and our report represents one such case. In genitourinary tuberculosis, it is prudent to keep in mind that unusual presentations are common so as not to be misled.

\section{References}

1. Dounis A, Gow JG (1979) Bladder augmentation. A long-term review. Br J Urol 51:264

2. Psihrams KE, Donahoe PK (1986) Primary genitourinary tuberculosis-rapid progression and tissue destruction during treatment. J Urol 135:1033

3. Goel A, Seth A, Kumar R (2002) Autocystectomy following extensive genitourinary tuberculosis: presentation and management. Int Urol Nephrol 34:325-327

4. Ba-Thike K, Than-Aye, Nan-Oo (1992) Tuberculous vesicovaginal fistula. Int J Gynaecol Obstet 37:127-130

5. Singh A, Fazal AR, Sinha SK, Ambasta SS, Kulshreshta (1988) Tuberculous vesicovaginal fistula in a child. Br J Urol 62:615 